\title{
Event-Related Potential Correlates of Long-Term Memory for Briefly Presented Faces
}

\author{
Carrie A. Joyce and Marta Kutas
}

\begin{abstract}
Electrophysiological studies have investigated the nature of face recognition in a variety of paradigms; some have contrasted famous and novel faces in explicit memory paradigms, others have repeated faces to examine implicit memory/ priming. If the general finding that implicit memory can last for up to several months also holds for novel faces, a reliable measure of it could have practical application for eyewitness testimony, given that explicit measures of eyewitness memory have at times proven fallible. The current study aimed to
\end{abstract}

\section{INTRODUCTION}

Many studies have investigated the nature of face recognition using various methodologies and experimental designs. Some have contrasted famous versus novel faces to investigate explicit memory processes, whereas others have used closely spaced repetitions of famous or novel faces to investigate priming processes (e.g., Bruce, Burton, Carson, Hanna, \& Mason, 1994).

Although indirect, nonconscious forms of memory have been found to last for even up to 16 months after initial exposure (e.g., Sloman, Hayman, Ohta, Law, \& Tulving, 1988), to our knowledge, no one has examined long-term implicit memory for novel faces. Because implicit memory is often more resilient than explicit memory to factors such as study duration (e.g., Von Hippel \& Hawkins, 1994; Kim \& Glanzer, 1993; Reynolds \& Pezdek, 1992; Dodson \& Reisberg, 1991; Musen, 1991; Read, Vokey, \& Hammersley, 1990), evidence of reliable, long-term priming for novel faces could have important practical implications. For instance, implicit memory may prove more reliable in eyewitness testimony. The legal system currently relies on long-term explicit retention after a single exposure to a novel face (i.e., eyewitness memory), even though it has been shown that this type of memory is quite malleable in a way that often results in forgetting and misidentification (e.g., Zaragoza \& Lane, 1994; Podd, 1990; Loftus \& Palmer, 1974).

Indirect measures of memory include response times (e.g., Sloman et al., 1988) and event-related brain po-

University of California determine whether indirect behavioral and electrophysiological measures might yield reliable estimates of face memory over longer intervals than have typically been obtained with priming manipulations. Participants were shown 192 faces and then tested for recognition at four test delays ranging from immediately up to 1 week later. Three event-related brain potential components (e.g., N250r, N400f, and LPC) varied with memory measures although only the N250r varied regardless of explicit recognition, that is, with both repetition and recognition. tentials (ERPs; e.g., Paller, Hutson, Miller, \& Boehm, 2003; Paller \& Gross, 1998) where covert memory is inferred from improved performance/decreased reaction times, and changes in electrophysiological responses correlated with a memory task although not with conscious recollection. To date, no experiments have directly examined these measures in studies of long-term retention of novel faces.

ERPs in particular provide a reasonable starting point for explorations of covert face memory. Their high temporal resolution affords a level of precision necessary for teasing apart early perceptually sensitive, implicit memory effects from later semantically sensitive, explicit memory effects. Furthermore, this methodology has been used to analyze explicit and implicit memory, thereby providing several candidate components (e.g., N250r: Pfütze, Sommer, \& Schweinberger, 2002; Begleiter, Porjesz, \& Wang, 1995; N400f: Bentin \& Deouell, 2000; Eimer, 2000; Paller, Gonsalves, Grabowecky, Bozic, \& Yamada, 2000; LPC: Barrett, Rugg, \& Perrett, 1988; Bentin \& McCarthy, 1994).

One of the most consistent components described in the face-processing literature is the N170, a negativegoing potential peaking at $\sim 170$ msec at occipitotemporal electrode sites. The N170 and its positive counterpart, the vertex positive potential (VPP), are larger for faces than other classes of objects (e.g., Eimer, 2000; Schendan, Ganis, \& Kutas, 1998; Bentin, Allison, Puce, Perez, \& McCarthy, 1996; Jeffreys, 1996), are sensitive to whether faces are attended or not (Eimer, 2000), and are affected by stimulus features, such as spatial frequency (Goffaux, Gauthier, \& Rossion, 2003). 
The N170/VPP complex, however, does not vary with familiarity (Bentin \& Deouell, 2000; Eimer, 2000; Rossion et al., 1999) and thus is not expected to be useful for present purposes.

Another relatively early component elicited by faces, the N250 repetition effect (N250r), does vary with memory; it is larger to familiar than unfamiliar faces over inferior temporal electrode sites, between 200 and 300 msec (Pfütze et al., 2002; Schweinberger, Pickering, Jentzsch, Burton, \& Kaufmann, 2002; Begleiter et al., 1995; Schweinberger, Pfütze, \& Sommer, 1995). To date, this effect has only been observed in repetition priming paradigms when repetitions are closely spaced (Schweinberger, Pickering, Burton, \& Kaufmann, 2002). It appears to be somewhat image independent, as it is reduced, but not extinguished, by repetitions of the same individual in a different image (Schweinberger Pickering, Jentzsch, et al., 2002).

Two later memory-related effects-the N400f and P300 - have also been reported. The N400f, a frontal negativity between $\sim 200$ and $500 \mathrm{msec}$, is larger to repeated or famous faces compared with novel ones (Bentin \& Deouell, 2000; Eimer, 2000). Bentin and Deouell (2000) hypothesized that this effect is associated with activation of semantic information involved in identifying familiar faces as it is modulated by famous faces but not by faces that are made artificially familiar in an experimental setting (Paller et al., 2000). Eimer (2000) further proposed that the N400f depends on task-relevant attentional processing as it was present when faces were attended (i.e., when detecting face repetitions) but not when the faces were ignored (i.e., detecting digits in alphanumeric strings).

The late positive component (P300/LPC), occurring between $\sim 300$ and $1000 \mathrm{msec}$, also shows an increase to familiar items compared with novel items (Henson et al., 2003; Münte et al., 1997; Schweinberger et al., 1995; Bentin \& McCarthy, 1994; Barrett et al., 1988). The LPC effect is largest over centroparietal electrode sites and its magnitude varies with task parameters (Paller et al., 2003; Paller \& Gross, 1998), the relative familiarity of the stimulus (Henson et al., 2003; Münte et al., 1997; Schweinberger et al., 1995; Bentin \& McCarthy, 1994; Barrett et al., 1988), and the delay between item repetitions (Rugg \& Nagy, 1989), among other factors. The repetition effect for unfamiliar faces is smaller than that to well known (famous/family) faces (Münte et al., 1997; Schweinberger et al., 1995; Bentin \& McCarthy, 1994; Barrett et al., 1988; see Henson et al., 2003, for equivalent repetition effects). Furthermore, although the LPC difference between ERPs to new and repeated items is on the order of $\sim 2.5-8 \mu \mathrm{V}$ in explicit tasks, it is about half the size, $\sim 1$ and $4 \mu \mathrm{V}$, in implicit tasks (Paller et al., 2003; Paller \& Gross, 1998). Finally, the LPC repetition effect diminishes as delay interval increases, for example, decreasing by half when delay increases from $\sim 1-25 \mathrm{sec}$ to 45 min. (Rugg \& Nagy, 1989).
Although the N250r, N400f, and P300/LPC each show some promise as an indirect measure of long-term memory for faces, there are some issues to keep in mind. First, it has proven difficult to obtain reliable ERP repetition effects to nonfamous faces when repetitions are not immediate or in close temporal proximity (e.g., Paller, Bozic, Ranganath, Grabowecky, \& Yamada, 1999). Most studies use short repetition lags or compare responses to famous and nonfamous faces. None have examined repetition effects for unfamiliar faces at long lags (hours and days). Furthermore, few have systematically manipulated factors known to differentially affect implicit and explicit memory, such as encoding time. Such manipulations are critical to understanding different memory phenomena and how they relate to the ERP repetition effects.

The current study was designed to address some of these issues. First, we sought a long-lasting ERP index of single-trial face learning that was not contingent on explicit face recognition. We would expect such an index to occur coincident with or soon after early visual and structural encoding stages, possibly near the N250r. To this end, ERPs were recorded from the same individuals during multiple recognition tests (at half-hour, 1-hr, 1-day, and 1-week delays) following single-trial exposure to many (192) different faces. In an attempt to tease apart implicit from explicit memory effects, encoding time was modulated: we expected that an implicit memory index would be less affected by encoding time than an explicit one. Based on prior reports, we expect fluctuations in explicit memory performance to be reflected in later ERP repetition effects (i.e., N400f, P300/LPC), and not in the earlier N250r. By creating a heavy memory load (many unfamiliar faces), modulating encoding time, and requiring long-term retention, we also aimed to test the idea that the different repetition effects reported in the literature reflect the engagement of different underlying memory processes, whose presence in any particular experiment is a function of the type of stimuli (famous, unfamiliar), memory load, and task requirements (Schweinberger \& Burton, 2003).

\section{RESULTS}

\section{Accuracy}

Accuracy data were subjected to a single-factor analysis of variance (ANOVA) with four levels of delay (immediate, $1 \mathrm{hr}, 1$ day, 1 week). Participants performed above chance at all test delays (chance $=0$ for hits minus false alarms). Recognition memory accuracy (hits + correct rejections) decreased over time, $F(3,45)=6.93, p<.003$ : it was better at the immediate test than at the 1-hr, $F(1,15)=23.69, p<.001$, and 1-week, $F(1,15)=12.56$, $p<.01$, delays. Recognition at 1 day was also better than at 1 week, $F(1,15)=6.80, p<.02$ (Table 1$)$. As seen in 
Table 1. Accuracy Data

\begin{tabular}{|c|c|c|c|c|c|c|c|}
\hline & \multirow{2}{*}{$\begin{array}{l}\% \text { Correct } \\
\text { (Hits + CRs) }\end{array}$} & \multirow[b]{2}{*}{$\%$ Hits } & \multirow[b]{2}{*}{$\% C R s$} & \multirow[b]{2}{*}{$\%$ FAs } & \multicolumn{2}{|c|}{$\%$ Hits } & \multirow{2}{*}{$\begin{array}{l}\% \text { Hits }-\% \text { FAs } \\
(\% \text { Over Chance })\end{array}$} \\
\hline & & & & & Long & Short & \\
\hline $30 \mathrm{~min}$ & $66.22(5.86)$ & $56.33(13.9)$ & $76.14(11.0)$ & $23.86(11.0)$ & $64.50(14.1)$ & $48.16(16.8)$ & $32.47(12.1)$ \\
\hline $1 \mathrm{hr}$ & $61.15(5.25)$ & $45.83(18.2)$ & $76.49(14.5)$ & $23.51(14.5)$ & $57.66(17.6)$ & $33.89(21.4)$ & $22.32(10.7)$ \\
\hline 1 day & $62.90(6.44)$ & $43.15(18.0)$ & 82.75 (12.9) & $17.24(12.9)$ & $52.92(19.8)$ & 33.28 (19.3) & $25.90(13.1)$ \\
\hline 1 week & $59.99(4.36)$ & 34.67 (15.9) & $85.51(11.0)$ & $14.48(11.0)$ & $42.83(17.2)$ & $26.60(16.3)$ & $20.19(8.7)$ \\
\hline
\end{tabular}

$\mathrm{CR}=$ correct rejection; $\mathrm{FA}=$ false alarm.

Table 1, this pattern is driven by changes in hits and not in correct rejections.

The number of false alarms likewise decreased over time. The largest decline occurred between $1 \mathrm{hr}$ and 1 day, $F(3,45)=10.56, p<.001$. There were more false alarms at the immediate and 1-hr tests than at either 1 day, Immediate $\times 1$ Day: $F(1,15)=7.94, p<.02$; 1 Hour $\times 1$ Day: $F(1,15)=11.23, p<.01$; or 1 week, Immediate $\times 1$ Week: $F(1,15)=22.45, p<.001$; 1 Hour $\times 1$ Week: $F(1,15)=16.86, p<.001$ (Table 1$)$.

Response bias $(\beta)$ also changed over time, $F(3,39)=$ $4.88, p<.01$ : the bias to say "new" was much stronger at 1 week than at $1 \mathrm{hr}, F(1,13)=20.69, p<.001$, or 1 day, $F(1.13)=8.67, p<.02 .^{1}$

Effect of study duration was examined using a twofactor ANOVA with four levels of delay and two levels of study duration (hits only). Accuracy was better for faces studied for longer durations, $F(3,45)=15.06, p<.0001$, regardless of test delay (Table 1 ).

\section{Event-Related Potentials}

Based on the observed peaks (Figure 1) and previous reports, mean amplitudes were measured in several time windows and submitted to separate ANOVAs: N1 (70-90), VPP (140-190), N250r (240-270), N400f (450-550), and P300/LPC (600-900). Multiple-comparison tests at $p<.05$ were used to analyze simple effects. Geisser-Greenhouse corrections were employed as needed.

Three multivariate ANOVAs were performed: (1) DLY: four levels of delay (immediate, $1 \mathrm{hr}, 1$ day, and 1 week), two levels of stimulus status (old vs. new), five levels of anterior-posterior electrode site (frontal, central, parietal, temporal, and occipital), and two levels of hemisphere (left vs. right), correct trials only; (2) RSP: two levels of stimulus status (old vs. new) and two levels of response (correct vs. incorrect), with the data collapsed across test delay; and (3) STDY: three levels of study time (long study vs. short study vs. new), for correct trials with the data collapsed across delay.

The first analysis (DLY) revealed which components were sensitive to memory status, and how long those memory effects lasted. The second analysis (RSP) distinguished components that were not sensitive to response, presumably reflecting implicit memory from those that were presumably reflecting explicit memory. The third analysis (STDY) distinguished components sensitive to study time manipulations (primarily explicit components) from those that were not (primarily implicit components). The minimum number of trials per condition was 16 (average $\sim 24$ for DLY and $\sim 32$ for RSP/ STDY).

Additional analyses were performed separately for each test delay. However, because of minimal bin counts (e.g., 3-4) there was not enough statistical power to obtain reliable results. No condition-related differences were found during the N1 or VPP windows. Thus, analyses for these intervals are not reported.

\section{N250r (240-270)}

There was a main effect of stimulus status on the N250r, RSP: $F(1,15)=6.86, p<.01$; old faces elicited more negative ERPs than new faces (Figure 2). Faces studied for longer intervals showed a larger N250r than those that were new or studied more briefly, STDY: $F(2,30)=$ $5.22, p<.01$ (Figure 4 ). There were no main effects of, nor interactions with, session or response. The negativity at anterior sites was larger than over posterior sites for all conditions, DLY: anterior-posterior electrode site $F(4,60)=254.79, p<.001$.

\section{N400f (450-550)}

From 450 to $500 \mathrm{msec}$ there was a main effect of delay, DLY: $F(3,45)=5.24, p<.002$; potentials at 1 day and 1 week were larger than those tested immediately or at $1 \mathrm{hr}$ (Figure 2). There was also a main effect of stimulus status, DLY: $F(1,15)=4.88, p<.03$, with new faces exhibiting more negative potentials than old faces (Figure 2). Furthermore, new faces and those studied for longer times elicited more negative peaks than faces studied more briefly, STDY: $F(2,30)=13.13, p<.0001$ (Figure 4). 
Figure 1. Morphology and topography of the N1, VPP, N250r, P300, N400f, and LPC components recorded during viewing of faces.

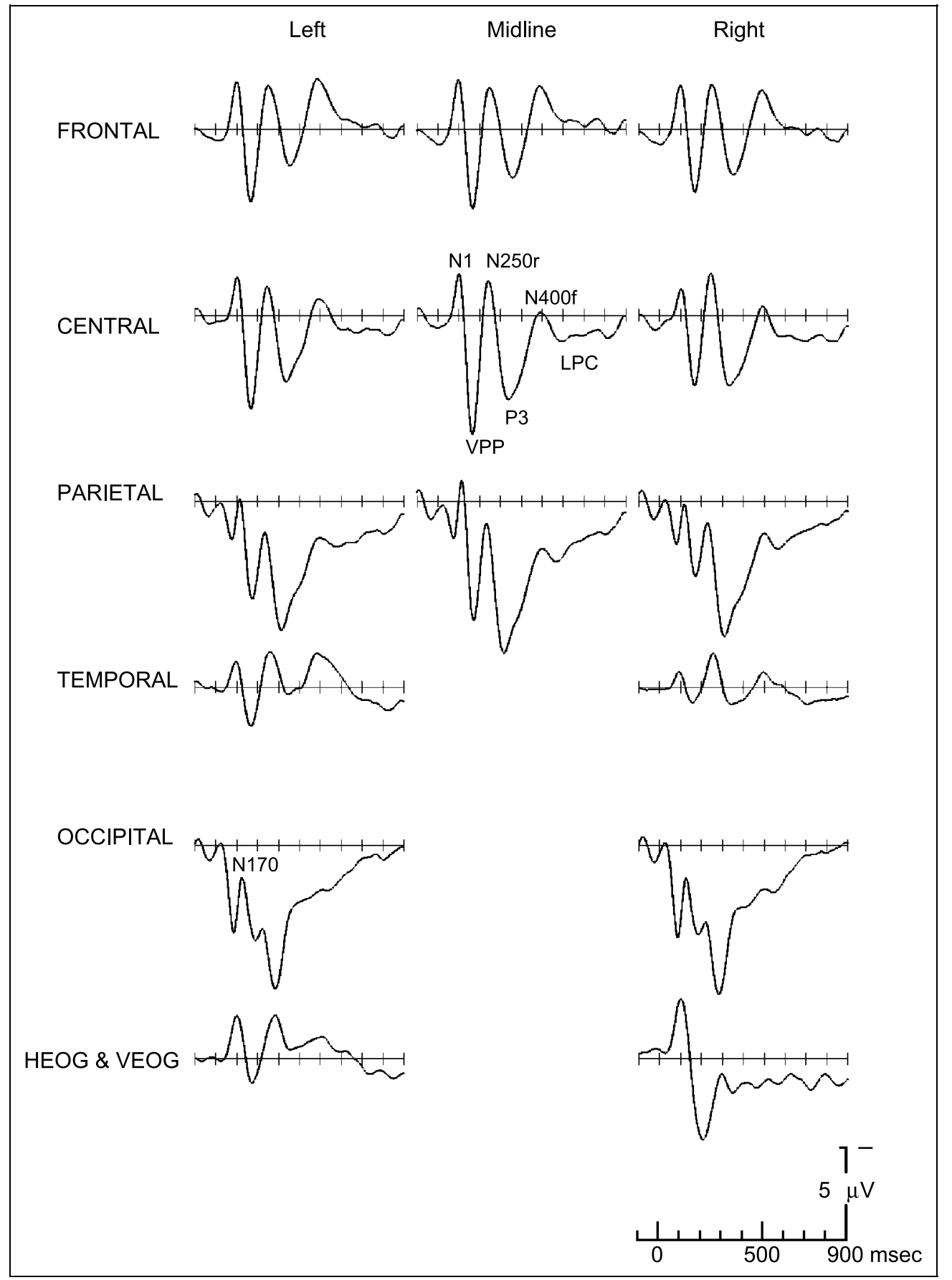

A main effect of anterior-posterior electrode site, DLY: $F(4,60]=98.33, p<.0001$, revealed larger potentials over anterior than posterior electrodes. This effect varied with delay, DLY: $F(12,180)=1.86, p<.04$, such that the difference between anterior and posterior electrodes was larger at the later test delays.

\section{LPC (600-900)}

Between 600 and 900 msec there was a main effect of delay, DLY: $F(3,45)=3.63, p<.02$; potentials during the immediate test were more positive than those observed at 1 day or at 1 week (Figure 2). Old faces exhibited more positive potentials than new faces, RSP: $F(1,15)=14.74, p=.001$ (Figure 2), and correctly identified faces exhibited more positive potentials than those incorrectly identified, RSP: $F(1,15)=6.32, p<.02$ (Figure 3). There was an interaction between stimulus status and response, RSP: $F(1,15)=5.48, p<.02$, whereby correctly identified old faces elicited larger positive potentials than incorrectly identified old faces and all new faces (Figure 3). There was also an interac- 
Figure 2. Mean amplitude ERPs to old (dotted) and new faces (solid) recorded at $\mathrm{F} 3, \mathrm{CZ}$, and $\mathrm{F} 4$ during the immediate, 1-hr, 1-day, and 1-week test delays. Correct trials only. Baselined to the N1 time window.

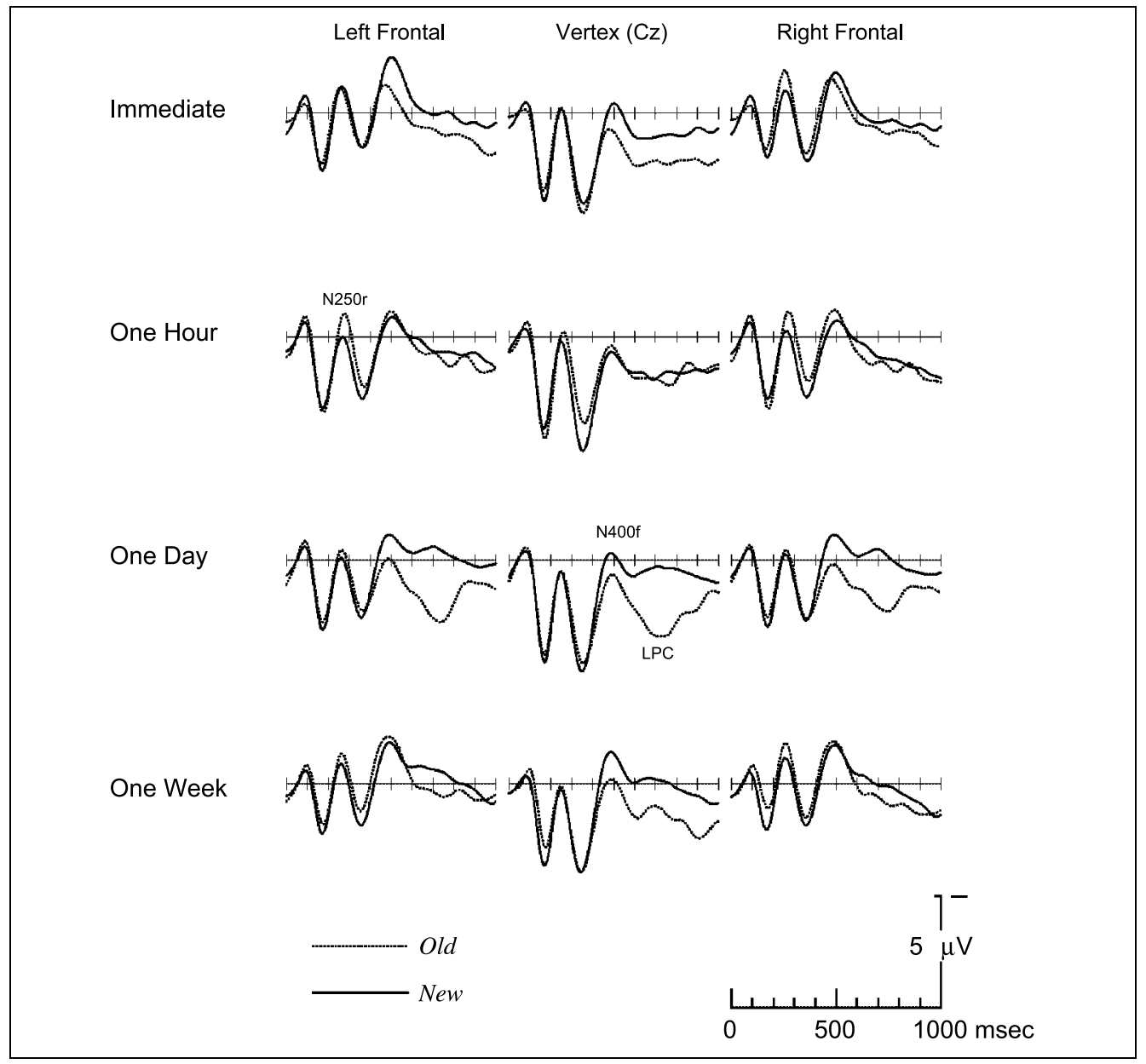

tion of stimulus status with delay, DLY: $F(3,30)=2.93$, $p<.04$; the old new difference was only significant at the 1-day and 1-week delays (Figure 2).

The LPC was also affected by study time, STDY: $F(2,30)=12.96, p<.003$ : it was largest for faces studied for short durations, intermediate for faces studied for longer durations, and smallest for new faces (Figure 4).

A main effect of anterior-posterior electrode site, DLY: $F(4,60)=16.68, p<.001$, revealed smaller potentials over frontal sites than over all other sites. Distributional differences did not interact with any of the experimental variables.

\section{DISCUSSION}

The present study was designed to find components of the ERP that would reflect a single prior exposure to a face whether or not the individual correctly recognized that face upon explicit testing. Five ERP components were analyzed, of which three were systematically related to some aspect of memory. No memory-related modulations were observed on the VPP component, consistent with prior reports (see also Jeffreys, 1996) or during the time window of the N1. There were, however, memory-related effects on (1) the N250r, a frontocentral negativity peaking at $\sim 250 \mathrm{msec}$ that was larger for old than new faces; (2) the N400f, a frontal negativity that was larger for new as compared with old faces; and (3) the LPC, a late, slow parietocentral positive shift that was larger for old than new faces. Of these, the traits of the N250r are most consistent with the desiderata for a brain component reflecting "implicit" recognition. Each of these will be discussed in turn.

\section{Frontocentral N250r and Perceptual Classification}

The N250r is arguably the most interesting repetition effect found in the present study with respect to the initial objective for it seems to provide evidence for onetrial learning of faces that is independent of explicit recognition memory. As in previous reports, the N250r was larger for old than new faces, although in prior work it was only observed with closely spaced repetitions (Pfütze et al., 2002; Schweinberger, Pickering, Jentzsch, et al., 2002; Begleiter et al., 1995; Schweinberger et al., 1995). Here, although there was no interaction of condition with delay, this repetition effect was largest 
Figure 3. Mean amplitude ERPS to old (dotted) and new (solid) faces that were either correctly (thick lines) or incorrectly (thin lines) identified by the subjects. Data are collapsed across test delay. Baselined to the N1 time window.

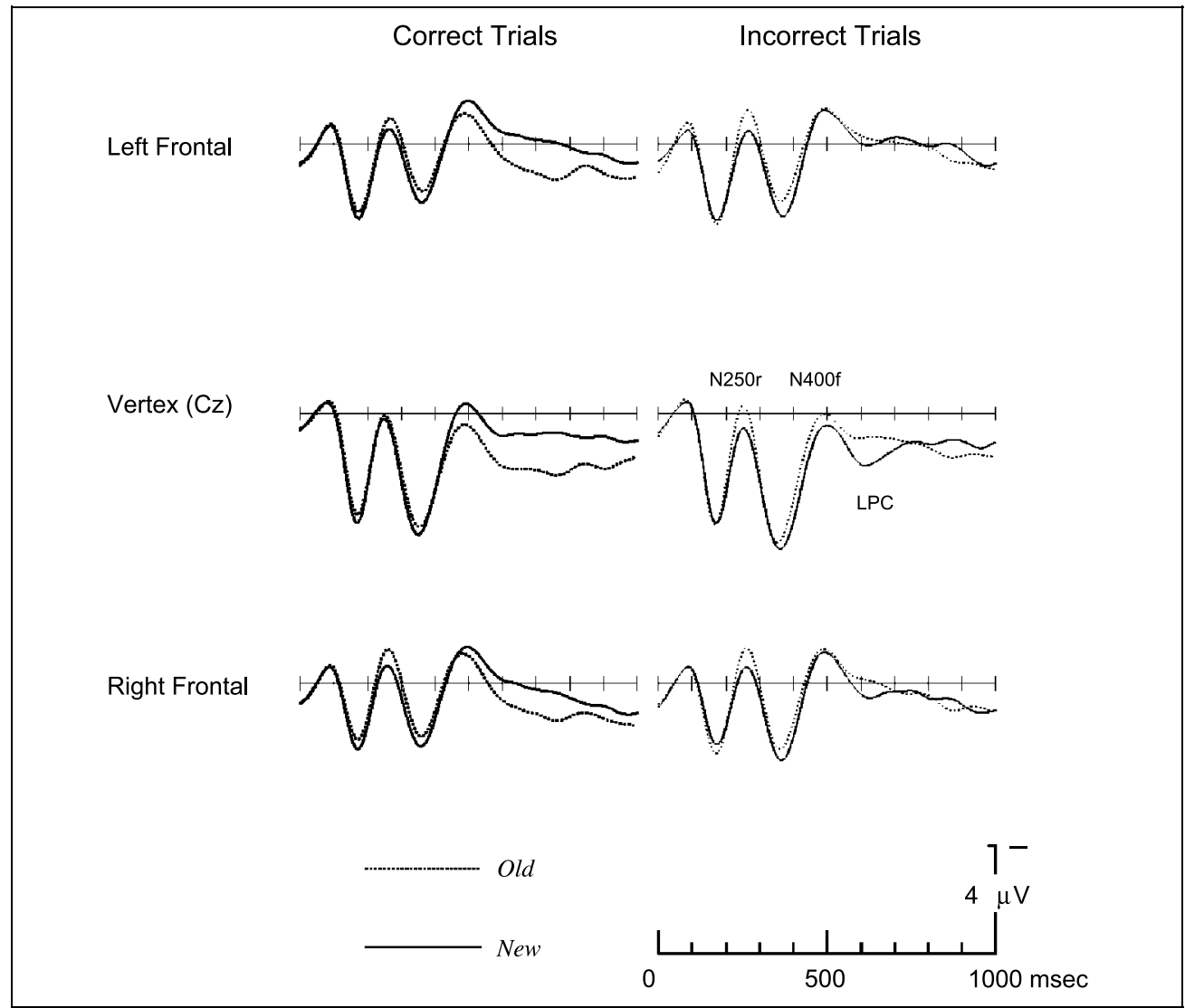

immediately and at $1 \mathrm{hr}$, and decreased markedly at later delays (although remaining visible at lateral sites). This pattern is somewhat consistent with short-lived priming effects, but other explanations are plausible. First, the number of stimuli in each condition at any given delay

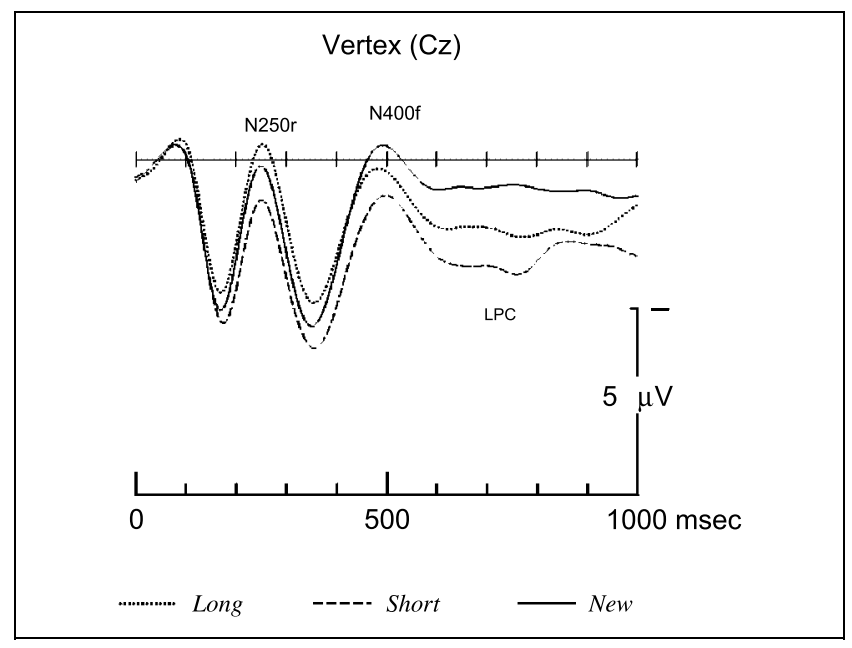

Figure 4. Mean amplitude ERPs to faces studied for long (dotted) and short (dashed) time intervals and to new faces (solid). Data from correct trials only, collapsed across test delay. Baselined to the N1 time window. was small. This was particularly problematic at later delays where correct recognition of old faces was relatively low. Thus, there was not enough statistical power to perform an accurate analysis of this effect at each test delay. Second, it may be that the few electrodes sampled in this study were not optimally located to record this component. Thus, at earlier delays when the signal was strongest, differences could be observed, whereas at later delays, when the signal was weaker, differences were attenuated. Along these same lines, it is not unusual for the orientation of dipole sources to change slightly over time. With only 13 scalp electrodes, there was not enough coverage to observe such changes, particularly when effects are small. These concerns will need to be addressed in additional experiments with more stimuli at each delay and a larger electrode array.

The N250r was sensitive to study duration, a factor that does not normally affect implicit memory. Here it may have played a greater role because participants were instructed to study the faces for a subsequent memory test, rather than being given an implicit task (e.g., famous vs. nonfamous) in which attention to a particular person's identity with an eye toward subsequent recognition is not crucial.

It has been suggested that the N250r reflects familiarity with a particular individual (Pfütze et al., 2002; Begleiter et al., 1995; Schweinberger et al., 1995). In 
the framework of the Bruce and Young (1986) model of face recognition, if the N170 corresponds to the "structural encoding" stage (Eimer, 2000; Bentin et al., 1996; Jeffreys, 1996), then the N250r might reflect the activity of the "face recognition units" (FRUs), a perceptual classification stage responsible for categorizing a face as familiar. Such a process need not be explicit, but could nonetheless benefit from longer exposure time during encoding. This hypothesis then might account for our finding that the repetition effect on the N250r is as large for old faces that participants fail to recognize as it is for old faces they successfully recognize, and larger for faces studied for longer than shorter durations.

A similar interpretation was offered by Paller et al. (2003) who found a negativity between 250 and 400 msec that was larger for repeated than new faces. Because explicit recognition scores were low, Paller et al. interpreted this effect as an index of perceptual priming. This effect was not observed when explicit recognition was good; instead, Paller et al. saw a later positivity. They suggested that explicit processes that occur in close temporal proximity to the perceptual priming effect tend to mask it unless explicit recognition is somehow experimentally suppressed. Unlike Paller et al., we observed the N250r for both explicitly recognized and unrecognized old faces, with no apparent interference by secondary explicit memory processes. We believe this is because the current paradigm allowed participants free movement of their eyes. This could serve to accelerate perceptual processing such that it resolves before the onset of later explicit memory processes. Consistent with this suggestion, the N250r effect occurs earlier ( 200-300 mecs), and is much shorter, with a sharper peak than that observed in Paller et al.

Henson et al. (2003) suggest a link between their observed decrease in hemodynamic activity to face repetitions in lateral midfusiform areas and the N250r and intracranial P290, thus placing the generator for the N250r in the ventral-temporal fusiform gyrus. However, although Puce, Allison, and McCarthy (1999) find semantic priming effects at the P290, they do not find any effects of familiarity, as seen in the current study with the N250r. In fact, Puce et al. find no effects of familiarity for any component in their study. Thus, if the generator for the N250r is in the lateral midfusiform gyrus and is associated with decreased activation found by Henson et al., it does not overlap with the face-specific ventral sites sampled by Puce et al. There are other areas that have also been implicated in the repetition of unfamiliar faces by intracranial recordings, such as the mid- and inferotemporal neocortex, orbitofrontal cortex, and the middle and inferior frontal gyri of the lateral frontal cortex (Seeck, Mainwaring, et al., 1997; Seeck, Michel, et al., 1997). Interestingly, these intracranial differences between repeated and nonrepeated faces appear even when explicit recognition is at chance (Seeck, Mainwaring, et al., 1997), similar to the response-independent
N250r. Our data cannot resolve these issues without replication and extension.

\section{The N400f and Familiarity}

The N400f, an enhanced negativity to new as compared with old faces, was observed at most test delays and was particularly large at the 1-day and 1-week tests. Although the effect of subject response was nonsignificant, visually this effect was larger for recognized than unrecognized faces (Figure 3).

Researchers have suggested that the N400f is related to the accessing of semantic information useful for face identification, the "person identity node" stage subsequent to perceptual classification by the FRUs in the Bruce and Young model (Bentin \& Deouell, 2000; Eimer, 2000). Given the dearth of identity-specific semantic information in the current study, combined with the sheer number of faces to be remembered, which undoubtedly hindered participants' ability to self-generate useful semantics, it seems unlikely that identity semantics per se could be responsible for the effect seen here.

Studies using other types of stimuli have proposed that the attenuation in N400 amplitude is an index of familiarity as opposed to overt recollection (Curran, 2000; Mecklinger, 2000; Rugg et al., 1998; Duzel et al., 1997, 2001). However, such familiarity effects have only been observed in paradigms using stimuli that either were familiar to the participant preexperimentally (e.g., words) or could be labeled verbally (e.g., scenes), conflating the influence of familiarity, recollection of preexperimental episodes, and verbal information processing (Yovel \& Paller, 2004).

The design of the current study does not support the verbal processing interpretation: participants were exposed to many unfamiliar faces, were given no labels of any kind, and had little opportunity to self-generate labels. However, the current design cannot distinguish between the effects of familiarity and recollection. It is possible that the N400f is large at long test delays for recognized old faces because a strong memory trace is required at these longer lags for recognition to take place. However, whether this trace relates to familiarity or overt recollection is unclear from this design. What is clear, however, is that recognized novel faces can elicit an N400f that distinguishes them from new faces, and the sensitivity of this component to response accuracy, albeit nonsignificant, seems to suggest that the N400f is in some way related to explicit memory.

Whereas explicit memory usually improves as study time increases, the N400f effect is larger for faces studied for shorter durations. However, this difference begins in the time window of the N250r and carries through the epoch; it is not generated at the N400f. If the data are baselined to the N250r, N400f study differences vanish (Figure 5). 
Figure 5. Mean amplitude ERPs to faces studied for long (dotted) and short (dashed) time intervals and to new faces (solid). Data from correct trials only, collapsed across test delay and rebaselined to the peak of the N250r.

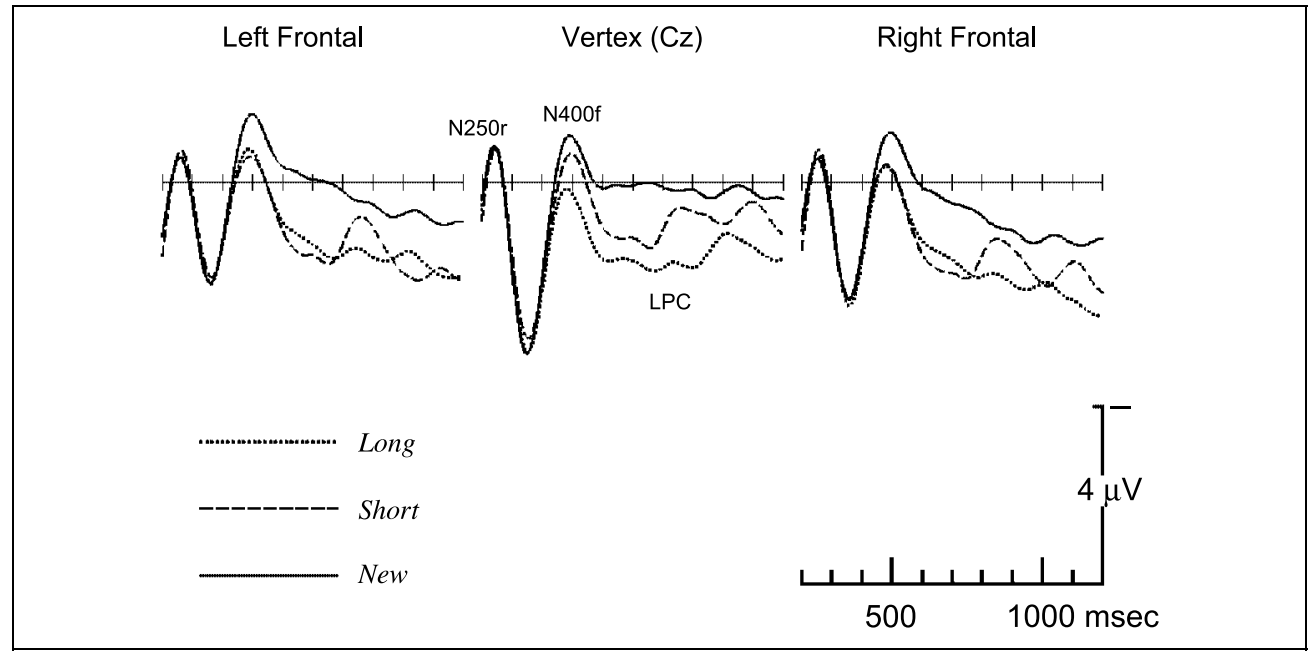

\section{The Centroparietal LPC and Consolidation}

The LPC, a late, slow centroparietal positive shift, larger for old than new faces, resembles later repetition effects reported for a variety of stimulus types including faces (Henson et al., 2003; Eimer, 2000; Münte et al., 1997; Schweinberger et al., 1995; Bentin \& McCarthy, 1994; Barrett et al., 1988). Consistent with previous reports, the LPC repetition effect was maximal over centroparietal electrode sites and present only for correctly recognized faces, suggesting a strong link to explicit memory processes. This interpretation coheres with the general finding that repetition effects at this latency are larger for familiar (famous/family) than unfamiliar faces (Schweinberger et al., 1995; Bentin \& McCarthy, 1994; Barrett et al., 1988) and for explicit than implicit memory tasks (Paller et al., 2003; Paller \& Gross, 1998). Its presence in many modalities and to many different stimulus types suggests it is a general explicit memory mechanism.

Two LPC results in the current study do not seem to fit neatly with this idea, however. Whereas explicit memory tends to improve as study time increases, the LPC, like the N400f, appears larger for faces studied for shorter durations. As with the N400f, however, this effect of study duration is a spillover from the study-related difference beginning at the N250r. Baselining the data relative to the N250r eliminates study duration differences (Figure 5), while preserving old/new differences, $F(2,30)=12.96, p<.001$. This result is unsurprising given that both study durations are relatively short (3001000 vs. $1000-3000 \mathrm{msec}$ ) and such a slight change in study exposure time might not be expected to yield substantially different results, particularly at long test lags.

A second counterintuitive finding is the apparently larger old/new LPC effect at the later (1-day and 1-week tests) relative to earlier test delays. This result is at odds with behavioral recognition data that generally show a power curve decrement over time: memory drops sharp- ly over the first hour and then at a slower rate over the next several days (e.g., Ebbinghaus, 1964). Recall, however, that only correct trials were included in the current analysis and that the LPC effect is driven completely by correctly identified faces. One simple explanation, then, is that because participants have a strong bias to say that a face is "new" as test delay increases (i.e., decreasing false alarms and increasing miss rates over time), the memory trace must be quite strong, hence associated with explicit recollection, before participants respond "old." Thus, the LPC, which in previous work has been found to be recollection- rather than familiarity-based (e.g., Schloerscheidt \& Rugg, 2004; Rugg, Allan, \& Birch, 2000; Rugg et al., 1998), does not appear at earlier test delays, emerging later instead. The time course of this effect suggests that it may be linked to memory consolidation, a process that is thought to require time in order to firmly encode items into long-term memory. There is evidence that sleep may be critical for such consolidation to take place (e.g., Li, Wu, Shao, \& Liu, 1991; Pearlman \& Greenberg, 1973), although this result is not uncontested (e.g., Oniani, 1982). Such a theory is consistent with the increase in the LPC old/new effect over time.

\section{Conclusions}

Although more research is necessary to elucidate the nature of the mechanisms involved in long-term face recognition, the current results indicate that there are both explicit and implicit ERP old/new differences to novel faces.

One peripheral issue that warrants consideration when interpreting the current data is that participants freely viewed the face images rather than fixating a particular location on the screen during testing, as is typical of ERP studies. This procedural change could have affected the data in several ways. It could conceiv- 
ably change the timing of ERP components by allowing participants to immediately fixate the most informative regions of the image for the task at hand rather than forcing them to glean such information out of peripheral vision. This was discussed briefly in relation to the potential speeding of perceptual processes at the level of the N250r allowing for temporal separation between the earlier and later memory effects. It also obviates the need for a secondary task implicit in most if not all ERP studies, namely, that of suppressing eye movements. Without direct comparison to data from an identical design in which eye movements are restricted, it is impossible to assess the effect of this change. However, because we replicate many of the previously reported behavioral and ERP memory effects, we have reason to believe that we are studying the same processes.

In summary, the current study used multiple recognition tests at various delays after a single trial exposure to many unfamiliar faces to define an ERP correlate of longterm, one-shot learning. A candidate component, the N250r, varied with memory even when there was no explicit face recognition. However, its endurance across test delay could not be adequately analyzed and thus could benefit from follow-up experiments. In addition, two later components, the N400f and the LPC, that have been discussed extensively in the literature and linked to explicit memory processes, showed effects suggesting that over time they became more sensitive to the strength of the memory trace, possibly through the process of consolidation. The current study illustrates that testing memory at multiple delays can help to elucidate the underlying processes reflected by various memoryrelated ERP components. Furthermore, by successfully replicating previous results, this experiment shows that free-viewing paradigms can be used fruitfully to investigate memory effects in a somewhat more natural way, thus alleviating irrelevant processing requirements (e.g., inhibiting eye movements) that could attenuate, mask, or even alter the very processes under study.

\section{METHODS}

\section{Participants}

Participants were 16 individuals ( 9 women, 7 men) affiliated with the University of California, San Diego, between 18 and 32 years of age (mean $=24$ ). Fifteen were right-handed (two with left-handed family members) and one was left-handed. Participants were paid for participating in three 2- to 4-hr experimental sessions.

\section{Stimuli}

Stimuli were 396 photographs drawn from the FERET (Phillips, Wechsler, Huang, \& Rauss, 1998) and AR-Faces (Martinez \& Benavente, 1998) face databases. Images were grayscale and cropped so that the faces would be roughly the same size. Image processing was used to normalize brightness/sharpness across photos. All images were resized to $320 \times 480$ pixels for presentation on CRT.

The demographic makeup of the faces was as follows: 237 men, 147 women; 262 Caucasians, 52 Asians, 18 Africans, 52 Indians; 93 wearing glasses; and 59 with facial hair. Photographs were divided into 16 lists counterbalanced for demographics.

Four experimental sets, each using all 16 lists, were created. Eight lists were shown during the study phase, such that photos from four of those lists were shown for a relatively long duration (3000-5000 msec) and the photos from the other four were shown for a relatively short duration (300-1000 $\mathrm{msec})$ : this manipulation is known to have a larger affect on explicit than implicit memory measures and will aid in teasing apart if/how components reflect different aspects of memory. At each test delay, two of the lists shown at study (one from long exposure and one from short) were represented, along with two lists of novel faces. Each studied list appeared at only one test delay. All lists were used at least once in every experimental condition (long/short study, and new). The delay at which studied lists were tested varied across sets (i.e., in Set A List 5 was tested at the immediate delay, whereas in Set B List 5 was tested at the 1 -hr test delay).

\section{Stimulus Presentation}

Participants were seated $50 \mathrm{~cm}$ from a computer monitor. Because eye movements and ERPs were being recorded concurrently, participants' heads were stabilized via a customized dental palate bar.

In the first session, participants experienced a study phase followed by two memory tests, one within $\sim 20$ min of studying the faces (immediate) and another after about 1-hr delay. Between the immediate and the 1-hr tests, participants performed a filler task (reading and norming sentences). Participants were brought back for two additional recording sessions on the following day and a week later. In both of these sessions, participants' memory for the studied faces was tested. Photographs of faces (subtending visual angles of $34^{\circ}$ vertically and $22^{\circ}$ horizontally) were presented on a computer screen during all experimental phases.

During the study phase, a light gray box $\left(34^{\circ}\right.$ vertical $\times 22^{\circ}$ horizontal) with a black central fixation cross appeared for 1 sec followed by a photograph (order of short/long stimuli randomized). The next fixation/photo cycle began after $\sim 1-1.5$ sec.

During each test phase, the gray box with fixation was presented for $2 \mathrm{sec}$ with the fixation located at the top center of the fixation box (off the face) so as not to unduly influence where participants looked first. The photograph was then presented for $5 \mathrm{sec}$ followed by a representation of the top center fixation for 2 sec. 
Participants were asked to fixate both before and after the face to aid in registration of the eye movements. The next trial began $3 \mathrm{sec}$ after the second fixation.

\section{Procedure}

During the study phase, participants viewed 192 faces divided into 16 blocks (12 pictures each block, 96 short duration, 96 long duration) and were asked to study the faces so that they would be able to recognize them if they passed them later on the street.

For each test phase, eight blocks of 12 faces each were presented. Participants were asked to press one button if they recognized the face, and another if they did not, and to respond as quickly and accurately as possible. The responding (yes/no) hand was counterbalanced within each participant across test delays and across participants. There were four test phases: immediately (containing faces occurring in the preceding four blocks of the study phase) and after 1-hr (after the filler task), 1-day, and 1-week delays. In each test phase, participants saw 96 faces (48 studied: 24 long exposure, 24 short exposure; 48 new). Each face appeared in only one of the recognition tests.

\section{Recordings}

ERPs were recorded using tin electrodes at locations from the International 10-20 System (F3/F4, C3/C4, T3/ T4, P3/P4, O1/O2, Fz, Cz, Pz; Jasper, 1958) and referenced on-line to the left mastoid. Data were digitized at $500 \mathrm{~Hz}$ using a Nicolet (Madison, WI) SM2000 AC amplifier with an on-line band-pass filter from 0.016 to $100 \mathrm{~Hz}$ and a 60-Hz notch filter. After data collection, trials with drifting or blocked signals or excessive muscle artifact were eliminated from further analysis.

Because participants were allowed free movement of their eyes, it was necessary to perform artifact correction to eliminate electro-oculogram (EOG) noise before statistical analysis. Eye movements and blinks were corrected using an automated, independent components analysis (ICA)-based, heuristic algorithm (Joyce, Gorodnitsky, \& Kutas, 2004) in which the data are decomposed into their component parts using ICA, components containing eye movement contamination are eliminated, and the data are recombined from the remaining, eye-movement-free components. Finally, the data were re-referenced to the averaged mastoids.

\section{Acknowledgments}

The authors thank Ken Paller, Tim Curran, and two anonymous reviewers for comments on previous versions of this manuscript. This work was supported by NICHD (22614), NIA (08313), and the James S. McDonnell Foundation (15573-S6).

Reprint requests should be sent to Marta Kutas, Department of Cognitive Science, University of California, San Diego, 9500
Gilman Drive, San Diego, CA 92093-0515, or via e-mail: mkutas@ucsd.edu.

\section{Note}

1. The data of two participants were dropped from the bias analysis because they had no false alarms at one of the test delays.

\section{REFERENCES}

Barrett, S. E., Rugg, M. D., \& Perrett, D. I. (1988). Event-related potentials and the matching of familiar and unfamiliar faces. Neuropsychologia, 26, 105-117.

Begleiter, H., Porjesz, B., \& Wang, W. (1995). Event-related brain potentials differentiate priming and recognition to familiar and unfamiliar faces. Electroencephalography and Clinical Neurophysiology, 94, 41-49.

Bentin, S., Allison, T., Puce, A., Perez, E., \& McCarthy, G. (1996). Electrophysiological studies of face perception in humans. Journal of Cognitive Neuroscience, 8, 551-565.

Bentin, S., \& Deouell, L. Y. (2000). Structural encoding and identification in face processing: ERP evidence for separate mechanisms. Cognitive Neuropsychology, 17, 35-54.

Bentin, S., \& McCarthy, G. (1994). The effects of immediate stimulus repetition on reaction time and event-related potentials in tasks of different complexity. Journal of Experimental Psychology: Learning, Memory, \& Cognition, 20, 130-149.

Bruce, V., Burton, M., Carson, D., Hanna, E., \& Mason, O. (1994). Repetition priming in face recognition. In C. Umilta \& M. Moscovitch (Eds.), Attention \& performance 15: Conscious and nonconscious information processing (pp. 179-201). Cambridge: MIT Press.

Bruce, V., \& Young, A. (1986). Understanding face recognition. British Journal of Psychology, 77, 305-327.

Curran, T. (2000). Brain potentials of recollection and familiarity. Memory \& Cognition, 28, 923-938.

Dodson, C., \& Reisberg, D. (1991). Indirect testing of eyewitness memory: The (non)effect of misinformation. Bulletin of the Psychonomic Society, 29, 333-336.

Duzel, E., Cabeza, R., Picton, T. W., Yonelinas, A. P., Scheich, H., Heinze, H. J., \& Tulving, E. (1997). Task-related and item-related brain processes of memory retrieval. Proceedings of the National Academy of Sciences, U.S.A., 96, 1794-1799.

Duzel, E., Picton, T. W., Cabeza, R., Yonelinas, A. P., Scheich, H., Heinze, H. J., \& Tulving, E. (2001). Comparative electrophysiological and hemo-dynamic measures of neural activation during memory-retrieval. Human Brain Mapping, 13, 104-123.

Ebbinghaus, H. (1964). Memory: A contribution to experimental psychology. Oxford, UK: Dover.

Eimer, M. (2000). Event-related brain potentials distinguish processing stages involved in face perception and recognition. Clinical Neurophysiology, 111, 694-705.

Goffaux, V., Gauthier, I., \& Rossion, B. (2003). Spatial scale contribution to early visual differences between face and object processing. Cognitive Brain Research, 16, 416-424.

Henson, R. N., Goshen-Gottstein, Y., Ganel, T., Otten, L. J., Quayle, A., \& Rugg, M. D. (2003). Electrophysiological and haemodynamic correlates of face perception, recognition, and priming. Cerebral Cortex, 13, 793-805.

Jasper, H. H. (1958). The ten twenty system of the International Federation. Electroencephalography and Clinical Neurophysiology, 10, 371-375. 
Jeffreys, D. A. (1996). Evoked potential studies of face and object processing. Visual Cognition, 3, 1-38.

Joyce, C. A., Gorodnitsky, I. F., \& Kutas, M. (2004). Automatic removal of eye movement and blink artifacts from EEG data using blind component separation. Psychophysiology, 41, 313-325.

Kim, K., \& Glanzer, M. (1993). Speed versus accuracy instructions, study time, and the mirror effect. Journal of Experimental Psychology: Learning, Memory, E Cognition, 19, 638-652.

Li, D., Wu, S., Shao, D., \& Liu, S. (1991). The relationship of sleep to learning and memory. International Journal of Mental Health, 20, 41-47.

Loftus, E. F., \& Palmer, J. C. (1974). Reconstruction of automobile destruction: An example of the interaction between language and memory. Journal of Verbal Learning E Verbal Behavior, 13, 585-589.

Martinez, A. M., \& Benavente, R. (1998). The AR Faces database (CVC Tech. Rep. No. 24). West Lafayette, IN: Purdue University.

Mecklinger, A. (2000). Interfacing mind and brain: A neurocognitive model of recognition memory. Psychophysiology, 37, 565-582.

Münte, T. F., Brack, M., Grootheer, O., Wieringa, B. M., Matzke, M., \& Johannes, S. (1997). Event-related brain potentials to unfamiliar faces in explicit and implicit memory tasks. Neuroscience Research, 28, 223-233.

Musen, G. (1991). Effects of verbal labeling and exposure duration on implicit memory for visual patterns. Journal of Experimental Psychology: Learning, Memory, \& Cognition, 17, 954-962.

Oniani, T. N. (1982). Role of sleep in the regulation of learning and memory. Human Physiology, 8, 381-391.

Paller, K. A., Bozic, V. S., Ranganath, C., Grabowecky, M., \& Yamada, S. (1999). Brain waves following remembered faces index conscious recollection. Cognitive Brain Research, 7, 519-531.

Paller, K. A., Gonsalves, B., Grabowecky, M., Bozic, V. S., \& Yamada, S. (2000). Electrophysiological correlates of recollecting faces of known and unknown individuals. Neuroimage, 11, 98-110.

Paller, K. A., \& Gross, M. (1998). Brain potentials associated with perceptual priming vs. explicit remembering during the repetition of visual word-form. Neuropsychologia, 36, 559-571.

Paller, K. A., Hutson, C. A., Miller, B. B., \& Boehm, S. G. (2003). Neural manifestations of memory with and without awareness. Neuron, 38, 507-516.

Pearlman, C. A., \& Greenberg, R. (1973). Post-trial REM sleep: A critical period for consolidation of shuttle-box avoidance. Animal Learning \& Behavior, 1, 49-51.

Pfütze, E. M., Sommer, W., \& Schweinberger, S. R. (2002). Age-related slowing in face and name recognition: Evidence from event-related brain potentials. Psychology and Aging, 17, $140-160$.

Phillips, P. J., Wechsler, H., Huang, J., \& Rauss, P. J. (1998). The FERET database and evaluation procedure for face-recognition algorithms. Image and Vision Computing, 16, 295-306.

Podd, J. (1990). The effects of memory load and delay on facial recognition. Applied Cognitive Psychology, 4, 47-59.

Puce, A., Allison, T., \& McCarthy, G. (1999).

Electrophysiological studies of human face perception. III: Effects of top-down processing on face-specific potentials. Cerebral Cortex, 9, 445-458.

Read, J. D., Vokey, J. R., \& Hammersley, R. (1990). Changing photos of faces: Effects of exposure duration and photo similarity on recognition and the accuracy/confidence relationship. Journal of Experimental Psychology: Learning, Memory, \& Cognition, 16, 870-882.

Reynolds, J. K., \& Pezdek, K. (1992). Face recognition memory: The effects of exposure duration and encoding instruction. Applied Cognitive Psychology, 6, 279-292.

Rossion, B., Campanella, S., Gomez, C. M., Delinte, A., Debatisse, D., Liard, L., Dubois, S. Bruyer, R., Crommelinck, M., \& Guerit, J. M. (1999). Task modulation of brain activity related to familiar and unfamiliar face processing: An ERP study. Clinical Neurophysiology, 110, 449-462.

Rugg, M. D., Allan, K., \& Birch, M. D. (2000). Electrophysiological evidence for the modulation of retrieval orientation by depth of study processing. Journal of Cognitive Neuroscience, 12, 664-678.

Rugg, M. D., Mark, R. E., Walla, P., Schloerscheidt, A. M., Birch, C. S., \& Allan, K. (1998). Dissociation of the neural correlates of implicit and explicit memory. Nature, 392, 595-598.

Rugg, M. D., \& Nagy, M. E. (1989). Event-related potentials and recognition memory for words. Electroencephalography $\varepsilon$ Clinical Neurophysiology, 72, 395-406.

Schendan, H. E., Ganis, G., \& Kutas, M. (1998). Neurophysiological evidence for visual perceptual categorization of words and faces within $150 \mathrm{~ms}$. Psychophysiology, 35, 240-251.

Schloerscheidt, A. M., \& Rugg, M. D. (2004). The impact of change in stimulus format on the electrophysiological indices of recognition. Neuropsychologia, 42, 451-466.

Schweinberger, S. R., \& Burton, A. M. (2003). Covert recognition and the neural system for face processing. Cortex, 39, 9-30.

Schweinberger, S. R., Pfütze, E., \& Sommer, W. (1995). Repetition priming and associative priming of face recognition: Evidence from event-related potentials. Journal of Experimental Psychology: Learning, Memory, and Cognition, 21, 722-736.

Schweinberger, S. R., Pickering, E. C., Burton, A. M., \& Kaufmann, J. M. (2002). Human brain potential correlates of repetition priming in face and name recognition. Neuropsychologia, 40, 2057-2073.

Schweinberger, S. R., Pickering, E. C., Jentzsch, I., Burton, A. M., \& Kaufmann, J. M. (2002). Event-related brain potential evidence for a response of inferior temporal cortex to familiar face repetitions. Cognitive Brain Research, 14, 398-409.

Seeck, M., Mainwaring, N., Cosgrove, R., Blume, H., Dubuisson, D., Mesulam, M. M., \& Schomer, D. L. (1997). Neurophysiologic correlates of implicit face memory in intracranial visual evoked potentials. Neurology, 49, 1312-1316.

Seeck, M., Michel, C. M., Mainwaring, N., Cosgrove, R., Blume, H., Ives, J., Landis, T., \& Schomer, D. L. (1997). Evidence for rapid face recognition from human scalp and intracranial electrodes. NeuroReport, 8, 2749-2754.

Sloman, S. A., Hayman, C. A. G., Ohta, N., Law, J., \& Tulving, E. (1988). Forgetting in primed fragment completion. Journal of Experimental Psychology: Learning, Memory, \& Cognition, 14, 223-239.

Von Hippel, W., \& Hawkins, C. (1994). Stimulus exposure time and perceptual memory. Perception \& Psychophysics, 56, 525-535.

Yovel, G., \& Paller, K. (2004). The neural basis of the butcheron-the-bus phenomenon: When a face seems familiar but is not remembered. Neuroimage, 21, 789-800.

Zaragoza, M. S., \& Lane, S. M. (1994). Source misattributions and the suggestibility of eyewitness memory. Journal of Experimental Psychology: Learning, Memory, \& Cognition, 20, 934-945. 